HU-TFT-95-28

\title{
Neutrino conversions in hot plasma
}

\author{
KARI ENQVisT ${ }^{1}$, JUKKa MAALAMPI ${ }^{2}$ \\ Department of Physics, University of Helsinki, Finland \\ and \\ V.B. SEMIKOZ 3,4 \\ Department de Fisica Teorica, University of Valencia, Spain
}

September 26, 2021

\begin{abstract}
We discuss the excitation of sterile neutrinos in the early universe using general quantum kinetic equations, which also incorporate a possible primordial magnetic field $B$. We find a new contribution to the excitation propability, which has its origin in the shrinkage of the spin vector. In the absence of $B$ nucleosynthesis implies the constraint $\left|\Delta m^{2}\right| \sin ^{2} 2 \theta_{0} \lesssim 1.6 \times 10^{-6} \mathrm{eV}^{2}$ which is more restrictive than previous estimates. We also present examples of possible stringent limits for $B \neq 0$.
\end{abstract}

1 enqvist@phcu.helsinki.fi; ${ }^{2}$ maalampi@phcu.helsinki.fi; ${ }^{3}$ semikoz@evalvx.ific.uv.es ; ${ }^{4}$ On a leave of absence from the Institute for Terrestrial Magnetism, Ionosphere and Radio Wave Propagation, Russian Academy of Sciences, IZMIRAN,Troitsk, Russia. 


\section{Introduction.}

The recent observation of the cosmic microwave background temperature anisotropy on large scales by the COBE satellite has hinted towards the existence of a hot component in the dark matter (HDM) of the universe [1]. A light neutrino is an obvious candidate for hot dark matter. As is well known, there are a number of unrelated astrophysical and cosmological constraints on light neutrinos, which have to do with solar neutrinos and the deficit of muon neutrinos in the atmospheric neutrino fluxes [2]. Reconciling all these issues in the context of a three generation mixing model only is quite difficult, and as was first pointed out in [3], if neutrinos are to solve the dark matter, solar and atmospheric problems simultaneously, one actually has to introduce a fourth neutrino, which could well be a sterile neutrino. Moreover, it has been suggested [4] that the recently observed signals in the KARMEN detector could be explained by the the decay pattern $\pi^{+} \rightarrow \mu^{+} \nu_{s}$, where $\nu_{s}$ is light sterile neutrino. Simple extensions of the standard electroweak model which can accommodate all known hints for neutrino masses, including solar and atmospheric neutrino observations also postulate the existence of a light neutrino $\nu_{s}$ [5,6]. In some of these models such sterile neutrino is the HDM candidate [6]. (Sterile neutrino as warm dark matter has recently been discussed in [7].)

Very tight constraints on the neutrino mass matrix that includes a singlet $\nu_{s}$ can be deduced from the primordial nucleosynthesis bound on the excess relic energy density at the proton-neutron freeze-out, which takes place at $T \approx 0.7 \mathrm{MeV}$. The bound derives from the evaluations of the abundances of primordial ${ }^{4} \mathrm{He}$ and $\mathrm{D}$ and is usually quantified in units of relativistic neutrino species. For a recent discussion on the neutrino bound, see [ [ (see also [9]).

Because of the mixing, sterile neutrinos can be produced in weak collisions and thus be brought into equilibrium, whence they would count as an extra relativistic 
neutrino degree of freedom, in clear contradiction with the observations [10]. Equilibration can be avoided for a certain range of the mixing parameters, though. The region of the active-sterile neutrino oscillation parameters $\Delta m^{2}=m_{2}^{2}-m_{1}^{2}$ and $\sin ^{2} 2 \theta_{0}$ excluded by the Big Bang Nucleosynthesis (BBN) has been estimated to be [11]

$$
\begin{aligned}
& \left|\Delta m^{2}\right| \sin ^{4} 2 \theta_{0} \gtrsim 5 \times 10^{-6} \mathrm{eV}^{2}, \quad \nu_{a}=\nu_{e}, \\
& \left|\Delta m^{2}\right| \sin ^{4} 2 \theta_{0} \gtrsim 3 \times 10^{-6} \mathrm{eV}^{2}, \quad \nu_{a}=\nu_{\mu, \tau} .
\end{aligned}
$$

Folding in an actual nucleosynthesis code into neutrino evolution equations has verified this result [12].

Strong random magnetic fields change neutrino oscillations drastically. This is a pertinent issue because of the possibility that the observed galactic magnetic fields, which are of the order of microgauss, might have a primordial origin [13. If this is true, then the plasma of the early universe sustained enormous magnetic fields which may have affected also particle interactions. It is natural to assume that the primordial field is random. This is because any magnetic field is imprinted on the almost chaotically comoving plasma, which entangles and mixes the field lines. Recently it was pointed out [14] that in presence of such random magnetic fields the BBN constraints on sterile neutrino become more stringent than in isotropic plasma.

In the present paper we investigate the kinetics of the active-sterile neutrino conversions in hot plasma of the early universe using a set of quantum kinetic equations. (For earlier applications, see [10,11,15].) Our formalism is general and takes also into account quantum damping as well as the effects of randomly magnetized plasma. Apart from reproducing the earlier results obtained in particle approach, the kinetic approach reveals new effects, which give rise to a novel constraint on the neutrino mixing parameters. It turns out to be more stringent than the previous ones, which have been obtain ed from one-particle Schrödinger equation.

The organization of the paper is as follows. To describe neutrino propagation in hot plasma we derive, in Section 2, neutrino dispersion relations in magnetized 
plasma. We also discuss briefly the behaviour of random magnetic fields. In Section 3, starting from the quantum kinetic equations (QKE) first derived in [16], we obtain a general analytic solution for the neutrino conversion probability in equilibrium plasma at temperatures $T \gg m_{e}$. Special care is taken to include correctly the effects of a possible random backround magnetic field. In Section 4 we apply our general formalism to the special case of isotropic plasma. We find a new shrinking, aperiodic analytic solution for $\nu_{e} \rightarrow \nu_{s}$ neutrino conversions, which gives rise to a new,

stringent constraint on the mixing parameters. In Section 5 we include a primordial magnetic field and find for large fields a simple analytic expression for the conversion probability. The solution is a generalization of the one-particle result of [14].

In Section 6 we discuss the results and their applications both to neutrino physics and to the astrophysics of galactic magnetic fields.

\section{Neutrino propagation in hot plasma}

\subsection{Random magnetic fields}

In order to describe active-sterile neutrino oscillations in a general medium, which may also contain a random magnetic field, we need neutrino dispersion relations. We also need a model for the primordial magnetic field. Although there are a number of suggestions as to how a large magnetic field could arise in the early universe [17,18], we prefer a more phenomenological approach.

A primordial magnetic field, no matter what its origin, is imprinted on the comoving plasma, which in the early universe has a very large conductivity. We shall assume that the primordial plasma consist of elementary magnetic domains of size $L_{0}$. Within each such domain the magnetic field is taken to be uniform and constant, and the field in different domains is randomly aligned. For the root mean squared magnetic 
field $B_{r m s}=\sqrt{\left\langle\mathbf{B}^{2}\right\rangle}$, averaged over a volume $L^{3} \gg L_{0}^{3}$, we assume the scaling law

$$
B_{r m s}=B_{0}\left(\frac{T}{T_{0}}\right)^{2}\left(\frac{L_{0}}{L}\right)^{p}
$$

The temperature dependence of $B_{r m s}$ reflects simply magnetic flux conservation. How does $B_{\text {rms }}$ scale with distance is an unsolved issue and reflects our ignorance e.g. on the question as to how uncorrelated the fluxes in the neighbouring magnetic domains actually are. In the case of no correlation statistical averaging gives for the parameter $p$ the value $p=1 / 2$, whereas a random-walk argument yields the scaling $p=3 / 2$ [19.

Dissipation of $B$ was investigated in [19,20]. There it was found that the dissipation length at the recombination time is about $10^{10} \mathrm{~cm}$, which corresponds to $10^{10} \mathrm{~cm} \times\left(T_{r e c} / T_{B B N}\right) \simeq 2 \times 10^{4} \mathrm{~cm}$ at nucleosynthesis. If primordial magnetic fields are to be the seed field for the galactic dynamo [13], the primordial field should survive until recombination. This sets a limit for the size of the random magnetic field domain $L_{0}$ :

$$
L_{0} \geq L_{0}^{\min }=10^{3} \mathrm{~cm} \times\left(\frac{\mathrm{MeV}}{\mathrm{T}}\right)
$$

Primordial nucleosynthesis may be used to set limits on the size of $B$ 20,21.

For an uncorrelated random magnetic field the mean field $\left\langle B_{j}\right\rangle=0$, whereas

$$
\left\langle B_{i}(\mathbf{x}) B_{j}\left(\mathbf{x}^{\prime}\right)\right\rangle=\frac{1}{2 \lambda} \delta_{i j} \delta^{(3)}\left(\mathbf{x}-\mathbf{x}^{\prime}\right)
$$

Here the correlation length $\lambda$ is determined by the domain size $L_{0}$ and the value of root mean squared field $B_{\text {rms }}$ at the horizon scale $L=l_{H}(T)$ [19]:

$$
\begin{aligned}
& \frac{1}{\lambda}=\frac{3}{\pi(3-2 p)} B_{r m s}^{2}\left(l_{H}\right) L_{0}^{3}, \quad p \neq 3 / 2, \\
& \frac{1}{\lambda}=\frac{3}{\pi}\left(\ln \frac{l_{H}}{L_{0}}\right) B_{r m s}^{2}\left(l_{H}\right) L_{0}^{3}, \quad p=3 / 2 .
\end{aligned}
$$


The horizon scale defines a cut-off $k_{\max }$ for the wave number. Physically it corresponds to the scale of the inhomogeneity, $k_{\max }=2 \pi / L_{0}$ [19]. This is the reason for the appearance of the horizon size in the above formulas.

\subsection{Neutrino dispersion relations}

The ultrarelativistic dispersion of a standard electroweak neutrino in hot plasma depends on the neutrino interaction potential, which consists of two parts:

$$
V=V^{(v e c)}+V^{(a x i a l)}
$$

The potential is determined by the neutrino forward scattering amplitude off all particle species in the plasma, including magnetized charged leptons and antileptons. The vector interaction potential $V^{(v e c)}$ for a neutrino with a momentum $p=\langle p\rangle=$ $3.15 T$ is given by 22

$$
V^{v e c}=G_{F} \sqrt{2} n_{\gamma}\left[n_{\text {asym }}-A \frac{T^{2}}{M_{W}^{2}}\right],
$$

where $n_{\text {asym }}$ depends on the particle-antiparticle asymmetries in the plasma, normalized to the photon density $n_{\gamma}=0.244 T^{3}$, and $A \simeq 55$. In the hot primordial plasma with $T \gg m_{e}$, where particle asymmetries are small, the vector interaction potential $V_{\text {vec }}$ is dominated by the second, non-local term, which reads

$$
\left|V^{(v e c)}\right| \simeq 3.4 \times 10^{-20}\left(\frac{T}{\mathrm{MeV}}\right)^{5} \mathrm{MeV} .
$$

The axial potential $V_{\text {axial }}$ is present only if the plasma supports a magnetic field. It is given by [14]

$$
V^{(a x i a l)}=\mu_{e f f} \frac{\mathbf{k} \cdot \mathbf{B}}{k}+\frac{\mu_{e f f}^{2}}{2 k}\left(B^{2}-\frac{(\mathbf{k} \cdot \mathbf{B})^{2}}{k^{2}}\right) .
$$


Here the quantity $\mu_{\text {eff }}$ is defined by

$$
\mu_{e f f}=\frac{e G_{F}\left(-2 c_{A}\right) T \ln 2}{\sqrt{2} \pi^{2}} \simeq-12 c_{A} \times 10^{-13} \mu_{B}\left(\frac{T}{\mathrm{MeV}}\right)
$$

where $c_{A}=\mp 0.5$ is the axial coupling in the weak lepton current (the upper sign is for $\nu_{e}$, the lower one for $\left.\nu_{\mu, \tau}\right)$, and $\mu_{B}=e / 2 m_{e}$ is the Bohr magneton. In our case it is sufficient to consider only the first term of $V^{(\text {axial })}$ since according to eq. (2.1) $\mu_{e f f} B \ll k \sim 3 T$.

\section{Neutrino conversions in hot plasma}

\subsection{Averaging over random magnetic fields}

Quantum kinetic equations take into account the inherent quantum nature of neutrino oscillations ( [16] and references therein, see also [23]). Adopting this approach we now derive a general expression for the probability of active-sterile neutrino conversions in the early universe.

The time evolution of the system of the active neutrino $\nu_{e}$ and a sterile neutrino $\nu_{s}$ can be described in terms of a polarization vector $\left(P_{0}(t), \mathbf{P}(t)\right)$, whose z-component gives the the excess of $\nu_{s}$ over $\nu_{e}$ in the neutrino ensemble at a given moment of time. In Ref. [16] the general time evolution equations of the polarization vector were derived (eqs. (28) to (31) of [16]), and these are applicable for any neutrino transitions. In our case, where one of the neutrinos is sterile with no interaction with the background matter, these equations are considerably simplified.

We shall assume a hierarchy between the relaxation times of the spatial distribution along the neutrino trajectory, and the momentum distributions. This assumption allows us to factorize out the equilibrium momentum distribution $f_{\nu}(k)=$ $[\exp (k / T)+1]^{-1}$, so that the density matrix can be written as $(\hat{\mathbf{k}}$ is the unit vector 
in the direction of $\mathbf{k}$ )

$$
\rho_{\nu}(t, \mathbf{k})=\frac{1}{2}\left[P_{0}(t)+P_{i}(t, \hat{\mathbf{k}}) \sigma_{i}\right] f_{\nu}(k) .
$$

The distributions of the active neutrino $\nu_{e}$ and the sterile neutrino $\nu_{s}$ can be written as

$$
\begin{aligned}
& f_{\nu_{e}}(t, \mathbf{k})=\frac{1}{2}\left[P_{0}(t)+P_{z}(t, \hat{\mathbf{k}})\right] f_{\nu}(k), \\
& f_{\nu_{s}}(t, \mathbf{k})=\frac{1}{2}\left[P_{0}(t)-P_{z}(t, \hat{\mathbf{k}})\right] f_{\nu}(k) .
\end{aligned}
$$

Let us note that our definitions (3.1) and (3.2) are more general than those given in 16$]$.

There is a hierarchy of scales in our problem: $L_{0} \ll L_{W} \ll l_{H}$, where $L_{0}$ is the previously defined scale of elementary domains in the randomly distributed magnetic field, $L_{W}$ is the length scale of weak interactions and $l_{H}$ is the horizon scale. Therefore we should average our QKE over the random magnetic field distribution before integration over the momenta. Moreover, we are interested in the neutrino conversion probability at physical distance much larger than $L_{0}$. It is described by the mean value of the flavour space polarization vector $\left\langle P_{z}(t, \hat{\mathbf{k}})\right\rangle=P_{z}(t)$, which does not depend on the neutrino momentum.

As the temperature changes slowly as compared with the neutrino oscillation rate one can write, using factorization (3.2), the active-sterile evolution equations in the form [16]

$$
\begin{aligned}
\frac{d P_{0}(t)}{d t} & =R(t, k) / f_{\nu}(k), \\
\frac{d P_{x}(t, \hat{\mathbf{k}})}{d t} & =-V_{z}(t, \hat{\mathbf{k}}) P_{y}(t, \hat{\mathbf{k}})-D(t, k) P_{x}(t, \hat{\mathbf{k}}), \\
\frac{d P_{y}(t, \hat{\mathbf{k}})}{d t} & =V_{z}(t, \hat{\mathbf{k}}) P_{x}(t, \hat{\mathbf{k}})-P_{z}(t, \hat{\mathbf{k}}) V_{x}-D(t, k) P_{y}(t, \hat{\mathbf{k}}), \\
\frac{d P_{z}(t, \hat{\mathbf{k}})}{d t} & =V_{x} P_{y}(t, \hat{\mathbf{k}})+R(k, t) / f_{\nu}(k) .
\end{aligned}
$$

Here $R(k, t)$ is the rate of the annihilation processes $\left(\nu_{e} \bar{\nu}_{e} \leftrightarrow e^{+} e^{-}\right)$, the general form of which is given in [16]. The damping parameter $D(k, t)$ is determined by the inelastic- and elastic neutrino collisions. 
The neutrino interaction potential $V_{z}$ is given by

$$
V_{z}(t, \hat{\mathbf{k}})=V^{(v e c)}-\Delta \cos 2 \theta_{0}+\mu_{e f f} B_{\|}(t)
$$

where $V^{\text {vec }}$ is given by the eq. (2.6), the magnetic term is approximated from eq. (2.7), $\Delta=\left(m_{1}^{2}-m_{2}^{2}\right) / 2 k$, and $V_{x}=\Delta \sin 2 \theta_{0}$.

The system of eqs. (3.3) yields the following quite complicated integro-differential equation for $P_{z}$ :

$$
\begin{aligned}
& {\left[\ddot{P}_{z}(t)+V_{x}^{2} P_{z}(t)+\left(\dot{P}_{z}(t)-\frac{R(t)}{f_{\nu}}\right) D(t)\right] F(t)} \\
& +\left(V^{(v e c)}-\Delta \cos 2 \theta_{0}\right)^{2} \int_{0}^{t} d t_{1}\left[\frac{d P_{z}\left(t_{1}\right)}{d t_{1}}-\frac{R\left(t_{1}\right)}{f_{\nu}}\right] F\left(t_{1}\right) \\
& +\mu_{e f f}^{2} \int_{0}^{t} d t_{1} B_{\|}(t) B_{\|}\left(t_{1}\right)\left[\frac{d P_{z}\left(t_{1}\right)}{d t_{1}}-\frac{R\left(t_{1}\right)}{f_{\nu}}\right] F\left(t_{1}\right) \\
& +\left(V^{(v e c)}-\Delta \cos 2 \theta_{0}\right) \mu_{e f f}\left\{B_{\|}(t) \int_{0}^{t} d t_{1}\left(\frac{d P_{z}\left(t_{1}\right)}{d t_{1}}-\frac{R\left(t_{1}\right)}{f_{\nu}}\right) F\left(t_{1}\right)\right. \\
& \left.+\int_{0}^{t} d t_{1} B_{\|}\left(t_{1}\right)\left(\frac{d P_{z}\left(t_{1}\right)}{d t_{1}}-R\left(t_{1}\right) / f_{\nu}\right) F\left(t_{1}\right)\right\} \\
& =0
\end{aligned}
$$

where $F(t)=\exp \left(\int_{0}^{t} D\left(t^{\prime}\right) d t^{\prime}\right)$ (here we omit in all the arguments the momentum variable k.) In the case of a random magnetic field integration over the dimensions transversal to the neutrino pragation direction allows us to omit the terms linear in $B_{\|}$. For the product $B_{\|}(t) B_{\|}\left(t_{1}\right)$ we may use the approximate result [14] $\left\langle B_{\|}(t) B_{\|}\left(t_{1}\right)\right\rangle /\left\langle B_{\|}^{2}\right\rangle \sim L_{0} \delta\left(t-t_{1}\right)$, so that this term reduces to

$$
2 \Gamma\left[\frac{d P_{z}(t)}{d t}-R(t) / f_{\nu}\right] \exp \left(\int_{0}^{t} D\left(t_{2}\right) d t_{2}\right) .
$$

Here $\Gamma$ is the damping induced by the presence of a magnetic field. Using eq. (2.4) for $p \neq 3 / 2$ yields the following expression:

$$
\Gamma=\mu_{e f f}^{2}\left\langle B_{\|}^{2}\right\rangle L_{0}=\frac{3}{4 \pi(3-2 p)} \mu_{e f f}^{2} B_{r m s}^{2}\left(L=l_{H}\right) L_{0} .
$$

Differentiating eq. (3.8) with respect to time, and omitting the common exponential factor, we finally obtain the following third order differential equation: 


$$
\begin{aligned}
& f(k)\left[\frac{d^{3} P_{z}}{d t^{3}}+2(\Gamma+D(t, k)) \frac{d^{2} P_{z}}{d t^{2}}\right. \\
& \left.+\left(\Delta_{m}^{2}+2 \Gamma D(t, k)+D^{2}(t, k)+\frac{d D(t, k)}{d t}\right) \frac{d P_{z}}{d t}+D(t, k) V_{x}^{2} P_{z}\right] \\
& =R(t, k)\left[2 \Gamma D(t, k)+D^{2}(t, k)+\frac{d D(t, k)}{d t}+\left(V^{(v e c)}-\Delta \cos 2 \theta_{0}\right)^{2}\right] \\
& +2 \Gamma \frac{d R(t, k)}{d t}
\end{aligned}
$$

where $\Delta_{m}$ is the usual neutrino oscillation frequency in isotropic medium:

$$
\Delta_{m}=\left[\left(V^{(v e c)}-\Delta \cos 2 \theta_{0}\right)^{2}+V_{x}^{2}\right]^{1 / 2}
$$

\subsection{Master equation for active-sterile conversion}

Before neutrino decoupling, pair annihilation does not contribute to the factor $R$, but it contributes, like elastic neutrino collisions, to the collision damping coefficient $D(t, k)$. At fixed temperature $D$ does not depend on time. This allows us to rewrite the evolution equation of the active-sterile neutrino conversion probability

$$
P=P_{\nu_{e} \rightarrow \nu_{s}}=\frac{1}{2}\left[1-\frac{P_{z}(t)}{P_{0}(0)}\right]
$$

in the following simplified form:

$$
\frac{d^{3} P}{d t^{3}}+2\left(\Gamma+\Gamma_{W}\right) \frac{d^{2} P}{d t^{2}}+\left(\Delta_{m}^{2}+2 \Gamma \Gamma_{W}+\Gamma_{W 1}^{2}\right) \frac{d P}{d t}+\Gamma_{W} V_{x}^{2} P=\frac{\Gamma_{W} V_{x}^{2}}{2}
$$

Here we have averaged eq. (3.10) over the normalized momentum distribution, and used the notations

$$
\begin{aligned}
\Gamma_{W} & =\int d k f(k) D(k)=\langle D\rangle, \\
\Gamma_{W 1}^{2} & =\int d k f(k) D^{2}(k)=\left\langle D^{2}\right\rangle,
\end{aligned}
$$

where $d k=\Omega d^{3} k /(2 \pi)^{3}$. 
Eq. (3.13) is our master equation for the active-sterile neutrino conversion. The initial conditions are easily determined from the equations (3.3)-(3.4) and are given by

$$
P(0)=0, \quad \dot{P}(0)=0, \quad \ddot{P}(0)=\frac{V_{x}^{2}}{2} .
$$

The solution of eq. (3.13) is of the general form

$$
P(t)=\frac{1}{2}+C_{1} e^{k_{1} t}+C_{2} e^{k_{2} t}+C_{3} e^{k_{3} t}
$$

Here $k_{1,2,3}$ are the roots of the Cartan equation

$$
k^{3}+a_{2} k^{2}+a_{1} k+a_{0}=0
$$

where the constant coefficients read

$$
\begin{aligned}
& a_{2}=2\left(\Gamma+\Gamma_{W}\right), \\
& a_{1}=\Delta_{m}^{2}+2 \Gamma \Gamma_{W}+\Gamma_{W 1}^{2}, \\
& a_{0}=\Gamma_{W} V_{x}^{2} .
\end{aligned}
$$

The roots

$$
\begin{aligned}
& k_{1}=-\frac{2\left(\Gamma+\Gamma_{W}\right)}{3}+\left(s_{1}+s_{2}\right), \\
& k_{2}=-\frac{2\left(\Gamma+\Gamma_{W}\right)}{3}-\frac{1}{2}\left(s_{1}+s_{2}\right)+\frac{i \sqrt{3}}{2}\left(s_{1}-s_{2}\right), \\
& k_{3}=-\frac{2\left(\Gamma+\Gamma_{W}\right)}{3}-\frac{1}{2}\left(s_{1}+s_{2}\right)-\frac{i \sqrt{3}}{2}\left(s_{1}-s_{2}\right),
\end{aligned}
$$

can be found by solving the Cartan equation in the standard way so that $s_{1}=$ $\left(r+\sqrt{r^{2}+q^{3}}\right)^{1 / 3}, s_{2}=\left(r-\sqrt{r^{2}+q^{3}}\right)^{1 / 3}$ with $r=\left(a_{1} a_{2}-3 a_{0}\right) / 6-a_{2}^{3} / 27, q=$ $a_{1} / 3-a_{2}^{2} / 9$. The constants $C_{i}=C_{i}\left(k_{1}, k_{2}, k_{3}\right)$ in eq. (3.16) are determined from the initial conditions (3.15).

In the general case the roots $k_{i}$ are quite complicated, but in some special cases they reduce to a rather simple expression. For instance, in the absence of collisions one has $\Gamma_{W}=\Gamma_{W 1}^{2}=0$, so that eq. (3.13) can easily be integrated. The probability 
eq. (3.12) takes in this case the form:

$$
P(t)=\frac{V_{x}^{2}}{2 \Delta_{m}^{2}}\left\{1-\exp (-\Gamma t)\left[\cosh \left(\sqrt{\Gamma^{2}-\Delta_{m}^{2}} t\right)+\frac{\Gamma}{\sqrt{\Gamma^{2}-\Delta_{m}^{2}}} \sinh \left(\sqrt{\Gamma^{2}-\Delta_{m}^{2}}\right)\right]\right\} .
$$

This result, which here followed from the general kinetic approach, was previously derived in [14] by using a different method. One can also readily see from eq. (3.19) that in the absence of a magnetic field our approach gives rise to the standard MSW result for the neutrino conversion probability [24]:

$$
P(t)=\frac{V_{x}^{2}}{\Delta_{m}^{2}} \sin ^{2} \frac{\Delta_{m} t}{2}
$$

\section{Shrinkage of the flavour spin}

\subsection{Solution for $B=0$}

Let us first apply our general formalism to the simple case of vanishing magnetic field by setting $\Gamma=0$. This has been previously studied in the literature by using the one-particle approach. The new effect revealed by the quantum kinetic approach is the shrinkage of the flavour polarization vector, which results in a more stringent bound on the vacuum oscillation parameters than the previous ones. The effect was first discussed qualitatively by Stodolsky in 25.

We take $\Delta_{m} \simeq V^{(v e c)} \gg \Gamma_{W}$, i.e. we assume that the system is far from the MSW resonance. We then find

$$
\begin{gathered}
s_{1}=\frac{\Delta_{m}}{\sqrt{3}}\left(1+\frac{\Gamma_{W} \sqrt{3}}{3 \Delta_{m}}+\frac{\Gamma_{W 1}^{2}-\Gamma_{W}^{2}}{2 \Delta_{m}^{2}}-\frac{\Gamma_{W} V_{x}^{2} \sqrt{27}}{6 \Delta_{m}^{3}}+O\left(\left(\Gamma_{W} / \Delta_{m}\right)^{4}\right),\right. \\
s_{2}=-\frac{\Delta_{m}}{\sqrt{3}}\left(1-\frac{\Gamma_{W} \sqrt{3}}{3 \Delta_{m}}+\frac{\Gamma_{W 1}^{2}-\Gamma_{W}^{2}}{2 \Delta_{m}^{2}}+\frac{\Gamma_{W} V_{x}^{2} \sqrt{27}}{6 \Delta_{m}^{3}}+O\left(\left(\Gamma_{W} / \Delta_{m}\right)^{4}\right)\right) .
\end{gathered}
$$

We thus have $s_{1}+s_{2}=2 \Gamma_{W} / 3-\Gamma_{W}\left(V_{x}^{2} / \Delta_{m}^{2}\right)$ and $s_{1}-s_{2}=(2 / \sqrt{3}) \Delta_{m}\left(1+\left(\Gamma_{W 1}^{2}-\right.\right.$

$\left.\left.\Gamma_{W}^{2}\right) / 2 \Delta_{m}^{2}\right)$, where in the difference we can neglect the dispersion term $\left\langle D^{2}\right\rangle-\langle D\rangle^{2}=$ 
$\Gamma_{W 1}^{2}-\Gamma_{W}^{2}$. Substituting $s_{1}$ and $s_{2}$ into the roots $k_{i}$ given by eq. (3.18), we obtain $k_{1}=-\Gamma_{W}\left(V_{x} / \Delta_{m}\right)^{2}, \quad k_{2}=-\Gamma_{W}+i \Delta_{m}$ and $k_{3}=-\Gamma_{W}-i \Delta_{m}$. The transition probability (3.16) is then given by

$$
P_{\nu_{e} \rightarrow \nu_{s}}=\frac{1}{2}\left\{1-\exp \left(-\left(V_{x} / \Delta_{m}\right)^{2} \Gamma_{W} t\right)+\frac{V_{x}^{2}}{\Delta_{m}^{2}}\left[\exp \left(-\left(V_{x} / \Delta_{m}\right)^{2} \Gamma_{W} t\right)-e^{-\Gamma_{W} t} \cos \Delta_{m} t\right]\right\} .
$$

For times much larger than the collision time $\Gamma_{W}^{-1}$, but much less than the depolarization time

$$
t_{d}=\frac{\Delta_{m}^{2}}{V_{x}^{2}} \Gamma_{W}^{-1}
$$

neutrino harmonic oscillations die away and the probability for $\nu_{e} \rightarrow \nu_{s}$ conversion is aperiodic:

$$
P=\frac{1}{2}\left[1-\exp \left(-\frac{t}{t_{d}}\right)\right] \simeq \frac{t}{2 t_{d}} .
$$

This probability is small,

$$
P \simeq \frac{V_{x}^{2}}{2 \Delta_{m}^{2}} \Gamma_{W} t \ll 1
$$

but nevertheless the aperiodic term dominates over the contributions from the last two terms in the brackets in eq. (4.1).

The earlier result for the transition probability [11 reads,

$$
P=V_{x}^{2} / 2 \Delta_{m}^{2}=\frac{\sin ^{2} 2 \theta_{0}}{2\left(1-2 x \cos 2 \theta_{0}+x^{2}\right)},
$$

where $x$ is defined through

$$
\frac{T}{\mathrm{MeV}}=\left(\frac{10^{7} \times x \Delta m^{2}}{2 \mathrm{eV}^{2}}\right)^{1 / 6}
$$

As can be seen, our result eq. (4.3) differs from eq. (4.5) by an additional large factor $\Gamma_{W} t \sim \Gamma_{W} / H \sim(T / M e V)^{3}$. This is due to spin shrinkage which is manifest in the kinetic approach. 


\subsection{Heuristic derivation of spin shrinkage}

The result eq. (4.3) has been discussed earlier by Stodolsky [25], who also pointed out that a large conversion probability $P=1 / 2$ may never be reached because depolarization time $t_{d}$ could be very large, in our case larger than the age of universe. Depolarization time can be estimated in a simple manner by noting that flavour spin rotation turns the longitudinal part of the spin into transversal, and at each collision the transversal part is, in effect, wiped out. This results in a shrinkage of the spin vector, and as $t \rightarrow \infty, P_{\nu_{e} \rightarrow \nu_{s}}(t)$ approaches the value $1 / 2$. The length of the spin vector $\mathbf{P}$ gives the degree of coherence, and as $t \rightarrow \infty, \mathbf{P}$ vanishes, which corresponds to a completely mixed incoherent state.

Qualitatively one can see this by writing the QKE (3.3)-(3.6) in the familiar form 25]

$$
\frac{d \mathbf{P}}{d t}=\mathbf{V} \times \mathbf{P}-D \mathbf{P}_{\perp}
$$

where now $\mathbf{V}=V^{(v e c)} \hat{n}_{z}+V_{x} \hat{n}_{x}, D \sim \Gamma_{W}$ and the transversal spin is given by $P_{\perp} \sim P\left(V_{x} / V_{z}\right)$ with $V_{x}=\Delta \sin 2 \theta_{0}$. It then follows that

$$
\frac{d P^{2}}{d t}=-2 \Gamma_{W} P_{\perp}^{2}
$$

yielding a shrinkage rate $P^{-2} d P^{2} / d t=-\Gamma_{W} \times V_{x}^{2} /\left(V^{(v e c)}\right)^{2}$, in agreement with eq. (4.3). Thus, in the presence of collisions the one-particle matrix density

$$
\rho_{\nu}(t, k)=\frac{1}{2}\left[P_{0}(t)+P_{i}(t) \sigma_{i}\right] f_{\nu}(k)
$$

tends in the formal limit $t \rightarrow \infty$ to the unpolarized one because of the slow spiralling of the spin vector into the origin:

$$
\rho_{\nu}(t, k) \rightarrow \frac{1}{2} P_{0}(\infty) f_{\nu}(k)
$$

The outcome is a unique equilibrium point for the system, given by $P_{x}=P_{y}=P_{z}=0$, where the number of active neutrinos equals the number of sterile neutrinos. 


\subsection{Big bang nucleosynthesis constraint}

The aperiodic term modifies considerably the constraint on the neutrino mixing parameters. Let us look at this matter more closely by first recapitulating the derivation of the earlier result [11, and then presenting a similar deriva tion within our present approach.

The condition for non-equilibration of sterile neutrinos is $\Gamma_{s}=P \Gamma_{W} \lesssim H$, which implies the constraint

$$
\left(\frac{\Gamma_{s}}{H}\right)_{\max }=0.6 \sin ^{2} 2 \theta_{0} \times \sqrt{10^{7}\left|\Delta m^{2}\right| /\left(2 \mathrm{eV}^{2}\right)} f_{1}^{\max }(y) \lesssim 1
$$

where $y= \pm x$ depending on the sign of the mass difference $\Delta m^{2}=m_{1}^{2}-m_{2}^{2}$. The function $f_{1}(y)$, defined as

$$
f_{1}(y)=\frac{\sqrt{y}}{1+2 y \cos 2 \theta_{0}+y^{2}},
$$

is the product of the probability (4.5) and the ratio $\Gamma_{W} / H$. For a small mixing angle

it reaches the maximum value $f_{1}^{\max } \simeq 3 \sqrt{3} / 16$ at $y=\left(\sqrt{3+\cos ^{2} 2 \theta_{0}}-\cos 2 \theta_{0}\right) / 3$. Substituting these values into eq. (4.7) one obtains the following allowed region for the mixing parameters [1]:

$$
\left|\Delta m^{2}\right| \sin ^{4} 2 \theta_{0} \lesssim 5 \times 10^{-6} \mathrm{eV}^{2}
$$

For a typical mixing angle $\sin 2 \theta \simeq 0.1$ this yields $\left|\Delta m^{2}\right| \lesssim 5 \times 10^{-2} \mathrm{eV}^{2}$.

In our case, where the conversion probability is given by eq. (4.4), one obtains the constraint

$$
\left(\frac{\Gamma_{s}}{H}\right)_{\max }=2.5 \times 10^{6} \sin ^{2} 2 \theta_{0} \times \frac{\left|\Delta m^{2}\right|}{e V^{2}} f_{2}^{\max }(y) \lesssim 1
$$

where the function

$$
f_{2}(y)=\frac{y}{1+2 y \cos 2 \theta_{0}+y^{2}}
$$


has the maximum value $f_{2}^{\max }=1 / 4 \cos ^{2} \theta_{0}$ at $y=1$. Thus, in our case the allowed region is

$$
\left|\Delta m^{2}\right| \sin ^{2} 2 \theta_{0} \lesssim 1.6 \times 10^{-6} \mathrm{eV}^{2}
$$

One should notice the different $\sin 2 \theta_{0}$ dependence compared with the earlier result (4.9). This difference gives rise to a more stringent constraint on the neutrino mass in our case. For example, for $\sin 2 \theta_{0} \sim 0.1$ we find $\left|\Delta m^{2}\right| \lesssim 1.6 \times 10^{-4} \mathrm{eV}^{2}$. The old and the new constraints in the $\left(\Delta m^{2}, \sin 2 \theta_{0}\right)$-plane are depicted in the Figure.

\section{Constraints in the presence of a magnetic field}

\subsection{Conditions for large magnetic effects}

Let us now consider neutrino propagation in hot magnetized plasma as described by the master equation (3.13). In this section we will assume a non-vanishing magnetic damping $\Gamma$ in eq. (3.13), together with a non-vanishing quantum damping $\Gamma_{W}$. We assume that the neutrino system has not yet reached the MSW resonance region, so that the oscillation frequency in matter $\Delta_{m}$ can be approximated by $\Delta_{m} \simeq V^{(\text {vec })}$. Furthermore, we take $\Delta_{m}^{2} \gg \Gamma_{W}^{2}, \Gamma_{W 1}^{2}$. We also assume that the dominant plasma effect on neutrino prapagation is due to random magnetic fields as given by eq. (2.1). This is the case where the magnetic damping rate is much larger than the oscilla-

tion rate of neutrinos, $\Gamma \gg \Delta_{m}$, and simultaneously $\Delta_{m}^{2} / \Gamma \Gamma_{W} \ll 1$. With these approximations, we are able to solve the QKE (3.13) analytically.

Let us study more closely these approximations. We may note that the magnetic damping rate

$$
\Gamma=3 \mu_{e f f}^{2} B_{r m s}\left(L=l_{H}\right) L_{0} / 4 \pi(3-2 p)
$$


has a minimum value

$$
\Gamma \geq \Gamma_{\min }=\frac{3.5(2.3)^{2 p}}{\pi(3-2 p)} \times 10^{-12-16 p} \frac{B_{r m s}^{2}\left(L=l_{H}\right)}{10^{24} G}\left(\frac{T}{\mathrm{MeV}}\right)^{5+2 p}\left(\frac{B_{0}}{T_{0}^{2}}\right)^{2} \mathrm{MeV}
$$

which corresponds to the minimum value of the magnetic domain size $L_{0}^{\min }$, given in $(2.2)$.

Let us now make a further assumption. To illustrate the potential importance of the magnetic effects, we take $B_{0} \simeq T_{0}^{2}$, which we believe is the largest possible random field that can be supported by the plasma. An example of this kind of a situation is possibly provided by the electroweak phase transition, where it has been argued [17] that fluctuating Higgs field gradients could generate magnetic fields of the order of $T_{E W}^{2}$.

To see when magnetic effects are important, we compare magnetic damping with $\Gamma_{W} \simeq 4.0 G_{F}^{2} T^{5}$ and the oscillation frequency $\Delta_{m} \simeq V^{(\mathrm{vec})}$. Since $V^{(\mathrm{vec})} \gg \Gamma_{W}$, a sufficient condition for the domination of magnetic damping is $\Gamma_{\min }>V^{(\mathrm{vec})}$. It turns out that even more restrictive condition is the requirement $\Delta_{m}^{2} / 2 \Gamma_{\min } \Gamma_{W} \ll 1$. This condition implies the following range of validity of the approximations discussed above:

$$
\frac{T}{\mathrm{MeV}}>\frac{(3 \pi(3-2 p))^{\frac{1}{2 p}}}{2.3} \times 10^{8-\frac{7}{2 p}}
$$

We note in passing that if we discard the hypothesis of the relic origin of the galactic seed field, thereby allowing considerably smaller domain sizes than given in eq. (2.2), the conditions $\Gamma \gg \Delta_{m} \gg \Gamma_{W}, \Delta_{m}^{2} / 2 \Gamma \Gamma_{W} \ll 1$ can be fulfilled only for very small values of the index $p$, i.e. when the magnetic field is very close to a constant. Our analysis does not cover this case. 


\subsection{Application to primordial nucleosynthesis}

Assuming the validity of the approximations discussed in the previous subsection, we obtain for the roots (3.18) the following simple expressions:

$$
\begin{aligned}
& k_{1} \simeq-2 \Gamma-\Gamma_{W}+\Delta_{m}^{2} / 2 \Gamma \simeq-2 \Gamma, \\
& k_{2} \simeq-\Gamma_{W}-\frac{\Delta_{m}^{2}}{2 \Gamma} \simeq-\Gamma_{W} \\
& k_{3}=-\frac{V_{x}^{2}}{2 \Gamma}+\frac{\Delta_{m}^{4}}{16 \Gamma^{2} \Gamma_{W}} \simeq-\frac{V_{x}^{2}}{2 \Gamma} .
\end{aligned}
$$

In $k_{3}$ we have neglected the second term $\Delta_{m}^{4} / 16 \Gamma^{2} \Gamma_{W}$ as it is resonable to assume that $V_{x} \leq \Delta_{m}$. From eq. (5.3) one can then easily see that for $t \gg \Gamma_{W}^{-1}$ only the last term

in eq. (3.12), the one proportional to $C_{3} e^{k_{3} t}$, survives, so that the active-to-sterile neutrino conversion probability now takes the form

$$
P \simeq \frac{1}{2}\left[1-e^{-V_{x}^{2} t / 2 \Gamma}\right]
$$

In order to avoid a conflict with the primordial nucleosynthesis constraint, the production rate $\Gamma_{s}=P \Gamma_{W}$ of the sterile neutrinos should be smaller than the expansion rate $H \simeq t^{-1}$ of the universe in the temperature range $1 \mathrm{MeV} \lesssim \mathrm{T} \lesssim \mathrm{T}_{\mathrm{QCD}}$. When $T \lesssim 1 \mathrm{MeV}$, left chiral neutrinos are decoupled, and collisions can no longer excite sterile neutrinos. Above the QCD phase transition temperature $T_{\mathrm{QCD}}$ sterile neutrino production is harmless, because their number densities are subsequently suppressed by the relative heating of the interacting species. From eq. (5.2) we find that, within our approximative solution, nucleosynthesis constraint is relevant only if $p \lesssim 0.6$. If this is so, we obtain from eq. (5.4) the condition $\left|V_{x}\right| \lesssim 2 H \sqrt{\Gamma_{\min } / \Gamma_{W}}$. This can be translated into a constraint on the neutrino mixing parameters:

$$
\frac{\left|\Delta m^{2}\right|}{\mathrm{eV}^{2}} \sin 2 \theta_{0} \lesssim 8 \times 10^{-2-8 p} \times \frac{(2.3)^{p}}{\sqrt{(3-2 p)}}\left(\frac{T}{\mathrm{MeV}}\right)^{3+p} \quad(p \lesssim 0.6)
$$

Since this limit is more restrictive the lower the temperature is, we substitute into eq. (5.5) the lower bound of the validity range defined in eq. (5.2). In this way we 
obtain our final result

$$
\frac{\left|\Delta m^{2}\right|}{\mathrm{eV}^{2}} \sin 2 \theta_{0} \lesssim 2(3 \pi(3-2 p))^{\frac{1}{2 p}} \times 10^{\frac{33}{2}-\frac{21}{2 p}}
$$

This result is displayed for $p=0.5,0.4$ and 0.3 , together with the $B=0$ case, in the Figure.

We may note that the presence of a magnetic field implies a limit which is more stringent than if $B=0$. Naturally, the limit is also more model dependent. In particular, it very much depends on our assumption that the primordial field is the seed field for the galactic dynamo, which reflects itself in $\Gamma_{\min }$. Moreover, our results are valid only for rather weak scaling behaviour. For larger $p$, it is likely that the bound (5.6) would be much weaker. On the other hand, weak scaling is needed if primordial fields are to provide the seed field.

\section{Discussion and conclusions}

We have considered active-sterile neutrino conversions in hot plasma of the early universe. Our starting point is a general quantum kinetic equation, and in the collisionless limit we reproduce the results of [14]. The great advantage of the kinetic approach is that one is able to follow the evolution of the transition probability over any number of collisions. In particular, it allows us follow the chain of collisions over time scales of the order of the Hubble scale. This fact provided us with a new analytic solution which actually dominates the active-sterile conversion probability in isotropic plasma. It is related to the shrinkage of the flavour spin. Such behaviour is the combined effect of neutrino oscillations, which convert longitudinal spin components to transversal ones, and simultaneous damping of the transversal component by collisions. Qualitatively this effect, progressive decoherence, was discussed earlier in [25]. Here it was realized explicitly in the analytic solution (4.1). 
An analogous shrinkage regime exists for Dirac neutrino spin-flip in plasma with a large scale random magnetic field [26]. The physical reason of the shrinkage of the total real spin there is the same as in our case.

It is perhaps surprising that the inclusion of a magnetic field, which leads to an increase of the energy gap between active and sterile neutrino spectra (see eq. (2.5)), tightens the constraint on the neutrino mixing parameters. In fact, the transition probability does decrease in the presence of a magnetic field exactly because of the energy gap increase. However, in the random field case the averaged conversion rates depend also on the neutrino squared mass difference $\Delta m^{2}$ in a subtle way. While in the isotropic case there is a saturation of the conversion probability as a function of $\Delta m^{2}$ in the presence of a magnetic field there is a linear dependence upon $\Delta m^{2}$. This behaviour is reflected in the stronger mixing angle dependence.

Acknowledgements One of us (V.S.) acknowledges the high energy physics group of Department of Theoretical Physics and Research Institute for Theoretical Physics at Helsinki University for hospitality. We thank Alexander Rez for fruitful discussions. The work has been supported by the Academy of Finland. 


\section{References}

[1] G.F. Smoot et al., Astrophys. J. 396 (1992) L1-L5.

[2] For a review, see E. Bellotti in Proc. of the XXVII Int. Conf. on High Energy Physics, ed. by P.J. Bussey and I.G. Knowles (IOP Publishing, Bristol 1995) vol I, p. 237.

[3] K. Enqvist, K. Kainulainen and M. Thomson, Phys. Lett. B288 (1992) 145.

[4] V. Barger, R.J.N. Phillips and S. Sarkar, Rutherford preprint RAL-95-026, hep$\mathrm{ph} / 9503295$.

[5] J.T. Peltoniemi, D. Tomassini and J.W.F. Valle, Phys. Lett. B298 (1993) 383.

[6] J.T. Peltoniemi and J.W.F. Valle, Nucl. Phys. B406 (1993) 409.

[7] S. Dodelson and L.M. Widrow, Phys. Rev. Lett. 72 (1994) 17.

[8] K. Olive and G. Steigman, preprint OSU-TA-2/95, hep-ph/9502400.

[9] T.Walker, G.Steigman, D.N.Schramm, K.Olive, and H.Kang, Astrophys. J. 376 (1991) 51.

[10] K. Kainulainen, Phys. Lett. B224 (1990) 191; R. Barbieri and A. Dolgov, Phys. Lett. B237 (1990) 440; Nucl. Phys. B349 (1991) 742; K. Enqvist, K. Kainulainen and J. Maalampi, Phys. Lett. B249 (1990) 531.

[11] K. Enqvist, K. Kainulainen, and M.J. Thomson, Nucl. Phys. B373 (1992) 498.

[12] X. Shi, D.N. Schramm and B.D. Fields, Phys. Rev. D48 (1993) 2563.

[13] L. D. Landau and E.M. Lifshitz, Electrodynamics of Continuous Media (Pergamon, Oxford 1960); Ya.B. Zeldovich, A.A. Ruzmaikin and D.D. Sokoloff, Mag- 
netic Fields in Astrophysics (McGraw-Hill, New York, 1980); E.N. Parker, Cosmological Magnetic Fields (Oxford Univ. Press, Oxford, 1979); A.A. Ruzmaikin, A.A. Shukurov and D.D. Sokoloff, Magnetic Fields of Galaxies (Kluwer, Dordrecht, 1988).

[14] V. Semikoz and J.W.F.Valle, Nucl. Phys. B425 (1994) 651

[15] M.A. Rudzsky, Astrophys. Space Sci. 165 (1990) 65; G. Raffelt and G. Sigl, Astroparticle Physics, 1 (1993) 165.

[16] B.H.J. McKellar and M.J. Thomson, Phys. Rev. D49 (1994) 2710.

[17] T. Vachaspati, Phys. Lett. B265 (1991) 258; K. Enqvist and P. Olesen, Phys. Lett. B319 (1993) 178.

[18] K. Enqvist and P. Olesen, Phys. Lett. B329 (1994) 195.

[19] K. Enqvist, A.I. Rez and V.B. Semikoz, Nucl. Phys. B436 (1995) 49.

[20] B. Cheng, D.N. Schramm and J.W. Truran, Phys. Rev. D49 (1994) 5006.

[21] D. Grasso and H. Rubinstein, Astropart. Phys. 3 (195) 95.

[22] D. Nötzold and G. Raffelt, Nucl. Phys. B307 (1988) 924.

[23] A.I. Akhiezer and S.V. Peletminsky, Methods of Statistical Physics (Pergamon, Oxford, 1981)

[24] M. Mikheyev, A. Smirnov, Soviet J. Nucl. Phys. 42 (1986) 913; L. Wolfenstein, Phys. Rev. D17 (1978) 2369; ibid. D20 (1979) 2634.

[25] L. Stodolsky, Phys. Rev. D36 (1987) 2273.

[26] V. Semikoz, Valencia Preprint IFIC/93-32 (1993). 


\section{Figure Caption}

The nucleosynthesis constraint on the $\nu_{e}-\nu_{s}$ mixing parameters. (a) old result;

(b) new result, which includes the shrinkage of the spin vector. Shown are also the limits obtained in the presence of a primordial random magnetic field with the scaling parameter $p=0.3,0.4$ and 0.5 (solid lines). In all cases the allowed region is below the contour. 


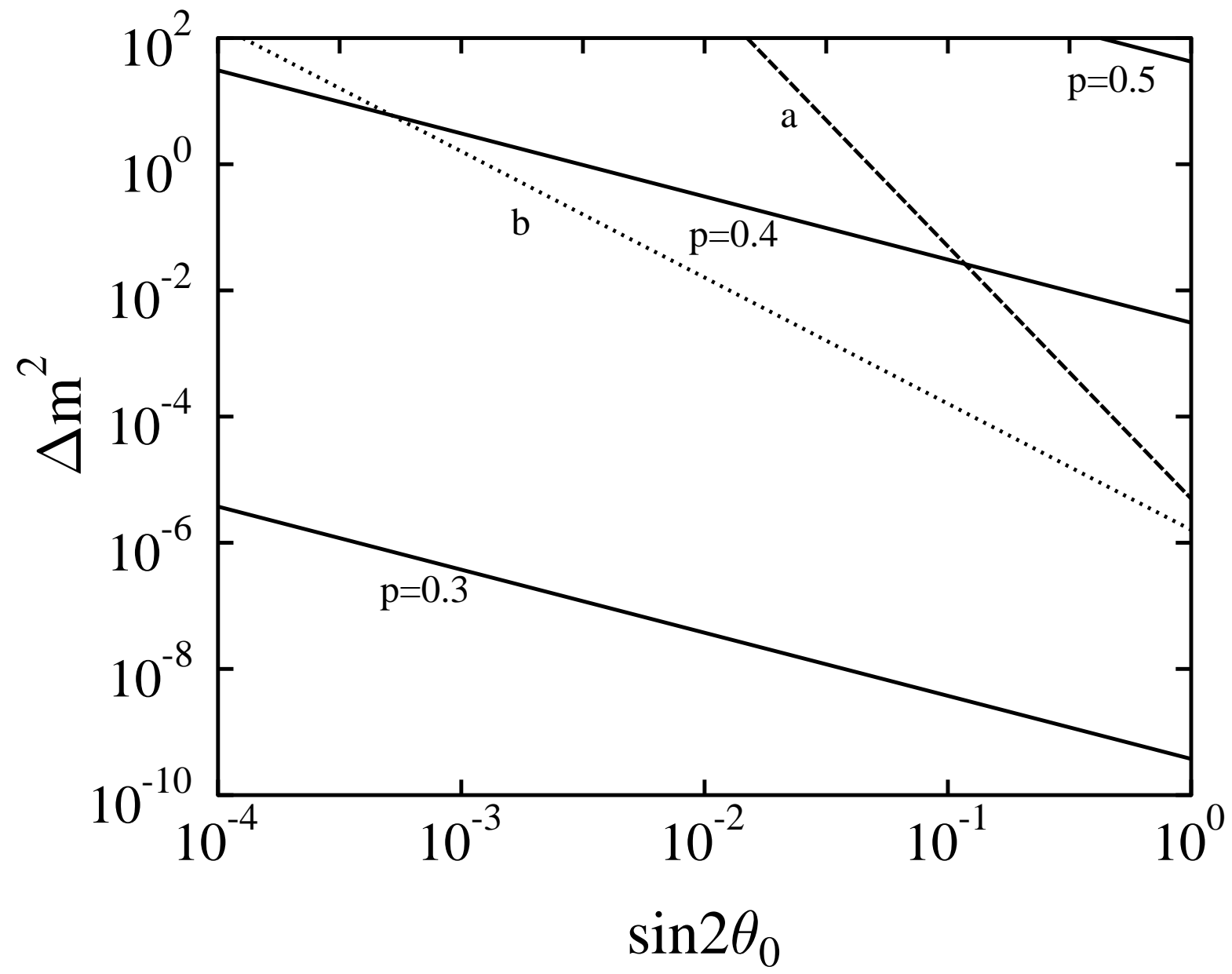

Figure 